My thanks are due to Professor D. M. Dunlop and Miss A. Buchan, who have taught and encouraged me in my work in the Dietetic Department of the Edinburgh Royal Infirmary, and to Miss A. Buchan and Dr A. P. Meiklejohn for advice in the preparation of this paper.

REFERENCES

Croom, J. H. (1950). Edinburgh med. $\not$. 57, 185 .

Lawrence, R. D. (195I). Lancet, 260, 1318.

Wilkerson, H. L. C. \& Krall, L. P. (1947). F. Amer. med. Ass. 135, 209.

\title{
Instruction of the Patient Attending the Dietetic Department of a Hospital
}

\section{By Ella ScotT, Senior Dietitian, The London Hospital, Whitechapel}

When a patient has to assume responsibility for his own diet, either as an outpatient or on discharge from hospital, the information given to him must be practical and easily understood. The dietitian must consider carefully the factors that will influence and help the patient to carry out the instructions. These factors apply both to a normal diet and to a therapeutic diet, which is, after all, only a modification of the normal.

\section{Factors to be understood before instructing the patient}

Income is most important, it controls the standard of living to a great extent and must therefore be given full consideration in relation to the diet. Unfortunately, food is usually the first essential to suffer restriction when the income is low or reduced. The nationality, racial habits and religious principles of the patient affect both the choice and preparation of the food and play a large part in deciding the eating habits of the individual. Home conditions influence the management of the diet. Space for storage of food, larder accommodation, cooking facilities and water supply all determine the selection and preparation of the food. The occupation and daily routine affect both calorie intake and the planning of meals. Extra rations of certain foods are allowed for men doing heavy manual labour, the agricultural worker is allowed an extra allowance of cheese. The hours of work may be in shifts. For the patient on a special diet this may mean detailed adjustments each week. Canteen facilities, time allowed for meals, and the distance between the home and the place of work may also determine the type of meal.

The dietitian must have a thorough knowledge of the current rations and of the available unrationed foods and of their cost. This is necessary when giving advice on either the normal or the therapeutic diet. In the latter instance it is essential to have a thorough knowledge of the regulations of the Ministry of Food governing the issue of special rations to those requiring therapeutic diets. Instructions contrary to the views and habits of the patients are seldom obeyed. 


\section{Instruction of the patient in hospital}

The majority of patients in hospital are greatly interested in food and in their meals. The stay in hospital offers an excellent opportunity for food education. A patient should leave hospital with new ideas on menu planning, on the serving of food and on balancing a diet.

The patient on a special diet, provided he is not too ill, commences his dietary instruction at the first interview with the dietitian. The object of this interview is to establish good relations between the patient and the dietitian, who, in the course of conversation, tactfully finds out any food preferences and dislikes, and emphasizes the importance of co-operation. This talk should, if possible, be carried out in an unhurried manner, an ideal difficult to achieve when precious time is slipping away and work is piling up. If a sense of hurry is allowed to creep into this interview many patients will hesitate, both then and later, to ask questions. As a result small but important dietetic problems often remain unsolved. Daily visits by the dietitian give opportunities for further instruction. Co-operation comes with understanding.

We all tend to be conservative in our food habits, which are a product of our upbringing and present circumstances. It is, however, well to realize that a diet that has to be more or less a permanency, as for the diabetic, can be planned to meet these habits to a large extent. One pitfall the dietitian must avoid is underestimation of the intelligence of her patient ; she must also avoid encouraging food fads and fancies by over-emphasizing likes and dislikes for particular foods. It should be suggested that presents of food, such as fruit and eggs, should be sent to the kitchen for inclusion in the diet and not allowed to remain in the locker and eaten as extras.

We have found that an individual menu slip, complete with the name of the patient and the type of diet, placed in a prominent position on the tray, stimulates interest in the meal and gives rise to many questions. It is interesting to note how many patients, particularly men, collect these slips for use when they go home.

The ambulant diabetic patient is encouraged to visit the diet kitchen. Here he learns how to plan his own meals using the daily menu, his own diet sheet and the alternative food list. He learns to write his own menu slips. We take this opportunity to demonstrate the use of homely measures and how to use a pair of scales. Simple tips are given, for example an ice cream scoup of mashed potato weighs $2 \mathrm{oz}$., and a slice of bread from a cut loaf weighs approximately I oz. These hints are helpful to those who will have to take meals in canteens or in restaurants. In this way the patient appreciates that his diet is well within his capabilities and his home circumstances.

\section{Instruction of the patient on discharge from hospital}

The final interview is used to check the diet, to clear up minor difficulties and, if necessary, to see the patient's relatives. With the diabetic it is wise to make sure 
that the scales, if these are supplied, are satisfactory. The nature of insulin reactions and the importance of carrying sugar or glucose tablets must be emphasized. The diabetic identity card should be filled in. In certain conditions a certificate for priority foods is given. The patients must be told then in detail how to obtain the recommended foods. It is often better to give instructions directly to the members of the family upon whom the patient is dependent for the preparation of his food, rather than to the patient. For example, the wife or mother of a patient with a permanent gastrostomy ought to visit the diet kitchen and be told the ingredients of, and the most satisfactory method of mixing, a highprotein, high-calorie mixture. This removes many doubts and fears, and proves that a complete diet can be produced in fluid form. We are proud of one patient who has had a gastrostomy for 4 years and continues to live a full and useful life. The diet has strayed a little from the original, but only in the non-essentials. A feeling of security is given when the patient and his family know that the dietitian can be consulted if difficulties arise.

\section{Instruction of the out-patient}

The out-patient derives the greatest benefit from individual instruction rather than from group discussion. Details of home circumstances, financial difficulties and other problems can be discussed more fully, and with less embarrassment, when there is no chance of being overheard. Many of the problems are simple and can be cleared up immediately, but others may need the help of the almoner or of the district nurse. Until the mind of the patient has been freed from worry about his medical condition, he cannot give full attention to his diet. The setting for the interview is important, a pleasant room with a friendly atmosphere is desirable. This, unfortunately, is not always available, and many have to rely on lowered voices for privacy.

Before any instruction is given the medical history should be studied for valuable information about the patient and his disease. This information is used to determine the best method of approach and to decide the amount of explanation necessary for understanding and co-operation on the part of the patient.

The diabetic patient must know something of the relationship of dietary treatment to insulin therapy, the reason for regular meals and why carbohydrate foods are controlled. The first interview must be carefully handled; only simple instruction should be given without the introduction of technical terms. The diet must be planned with the aid of the patient. Valuable help and support is given in many cases by the relative or friend accompanying the patient. Food models and pictures should be used to demonstrate the size of portions of food allowed in the diet, and specimens of household measures familiar to the patient in the home are useful when explaining the composition of the diet.

The dietitian should co-operate with the doctor in giving the certificate for any extra foods and should suggest how best they can be used. It may not be necessary to buy the full ration, and information on the value of margarine is welcomed by the diabetic patient who cannot afford to buy extra butter. 
In many diabetic clinics the almoner will arrange for the district nurse to give the insulin injections until the patient, or a relative, is able to do so, but the dietitian when required must be prepared to check the insulin dosage. A set of insulin syringes, and bottles for the different types and strengths of insulin, are all that is required. Since these bottles are only being used for teaching purposes, they are filled with water instead of insulin.

Our diabetic patients are given a pamphlet, Notes for the patient, to read at home. This gives details of the disease and of its treatment in simple language and stresses many of the points made at the interview. It also prepares the way for further instruction.

Insulin reactions, and the reasons for extra carbohydrate food when strenuous exercise is taken, are dealt with more fully at a second visit.

Suggestions for a light diet of the same food value, and for sandwiches to replace the midday meal, prove helpful in many cases.

Individual instruction can be supplemented by group discussion in the waiting room of the diabetic clinic. New recipes and variations in the diet can be demonstrated. It is not necessary to have a complete set of cooking equipment in order to do this. All that is required is a blackboard on which to write the recipe, a table, with plates and jugs containing the measured ingredients, and a specimen of the prepared food. A full explanation is given by the dietitian, or student dietitian, of measuring and weighing the ingredients and of the oven temperature and length of time required for cooking. Finally, each patient is taught how to fit the recipe into the dietary plan. Lists of tried recipes are available for patients on restricted-carbohydrate, on low-calorie, on low-sodium and on bland diets.

\section{Diets for the obese}

The overweight patient on a low-calorie diet attends the clinic at frequent intervals. Good results are obtained by careful explanation of a diet planned to show the patient what he may eat. Merely giving a list of forbidden and of permissible foods has not proved nearly so satisfactory. It is important to discuss the reason for dieting and the loss in weight to be expected in a given time. From the commencement of the treatment the patient should know that, once the weight reaches a satisfactory level, instruction will be given on how to keep it there.

Praise and encouragement for progress, or even a little mild bullying, are used in turn. The dietitian must have imagination as well as a wide knowledge of people if she is going to find a point of contact and be able to stimulate the patient to continue over a period of time. Weight cards, and weight graphs, with the target plainly marked, keep the interest alive. Demonstrations of portions of different foods with the same calorie value are both interesting and instructive. The diet is reviewed at each visit and, if weight loss is unsatisfactory, search must be made for ' hidden calories.' A visit to the local cinema may be instructive. How many of the audience does one see consuming potato crisps, peanuts and ice-cream? Amongst this audience may be a patient or potential patient. No question is too 
elementary to ask the patient; often an inquiry about sugar will produce the information that only glucose is used for sweetening purposes!

Foreign patients. Where many foreign patients have to be instructed, a knowledge of the different foods, customs and methods of cooking is essential in order to appreciate and simplify the difficulties. Special treats at holiday times and on feast days help to control the intake of extra food - a little leniency will often prevent overeating.

\section{Low-sodium diets}

The patient on a low-sodium diet needs constant help and encouragement to induce him to continue with the diet. Many everyday recipes can be adjusted and used to vary the monotony. Plain flour and salt-free margarine can be used for making shortbread and pastry ; foods can be fried in salt-free fat ; chives, parsley, onion and garlic can be used for flavouring with great success. Arrangements can be made with the local baker and grocer to supply salt-free bread and margarine on the presentation of a letter from the dietitian. Mistakes are easily made by the patient when trying to vary the diet. One patient, tired of eating salt-free bread, changed to home-made soda bread! Butter can be de-salted by those who dislike margarine. Printed recipes giving the methods are supplied.

\section{Diet for peptic ulceration}

The value of simple explanation is demonstrated when instructing the patient suffering from peptic ulceration. If the ulcer is likened to a sore on the hand the patient will readily appreciate the reason for bland, non-irritating foods. The necessity for small meals at frequent intervals can be brought home equally well.

\section{Antenatal clinics}

The therapeutic approach to dietetics is not applicable to work in the antenatal clinic, where the accent is on a balanced normal diet. A dietary history is taken to supply information on the quantity and kind of food usually eaten and on the amount of cooking that is done. It is useful to know if any of the meals are taken in a canteen or café. A simple form, with a list of relevant questions, serves as a guide. The patient's interest is aroused when she sees her intake of food written down. When faults are pointed out and reasoned improvements are suggested she is almost always ready to co-operate. Reasons should be given for the extra food allowances and for the vitamin supplements. On her first visit to the antenatal clinic each patient is seen by a dietitian. If the diet is not satisfactory the expectant mother attends regularly until it has improved.

In conclusion it cannot be too strongly emphasized that the success of instruction in therapeutic dietetics depends primarily upon a spirit of co-operation between patient and relatives on the one side and dietitian on the other. To achieve this a dietitian must have not only patience, perseverance, tact and imagination, but also an abiding interest in all human beings. 